\title{
Angle as a Fourth Fundamental Quantity
}

\author{
Jacques E. Romain*
}

(April 18, 1962)

\begin{abstract}
The advantage of considering angle as a fourth fundamental quantity of geometry and physics is stressed, and an alternative approach is suggested to introduce an angular dimension in such a way that the physical laws, in any form, are dimensionally homogeneous. A few examples are described to show how some equations of mathematics and mechanics should be revised to put the new point of view into practice.
\end{abstract}

\section{Introduction}

Chester H. Page ${ }^{1}$ recently wrote in this Journal a most interesting study about "physical entities and their mathematical representations." One of the ideas in that paper, which will be of interest here, is that advantage can be gained from considering angle as an additional fundamental quantity. Dr. Page rightly points out that, since the measure of an angle depends on the chosen angle unit, it would be natural not to consider it a dimensionless quantity. That point of view could hardly be overstressed, and the present writer has the unpleasant feeling of an artificial trick about the generally accepted rule that angle is a dimensionless quantity, but may only be measured in radians if trouble is to be avoided.

Moreover, ignoring the dimension of angle deprives dimensional analysis of a part of its fruitfulness. Although the number of fundamental quantities in a dimensional theory is not assigned by any logical prescription, it is well known ${ }^{2}$ that the optimum number of fundamental quantities is the smallest number that is enough to express every physical quantity in the theory in terms of properly chosen fundamental quantities, without arbitrarily assigning a dimensionless status to any physical constant. In view of these arguments, it seems rather surprising that earlier attempts ${ }^{3,4,5,6}$ did not seem to meet any widespread audience.

This paper describes an alternative approach to the introduction of an angular dimension. It must not be construed as polemics against Page's approach: actually both systems are equally consistent. However, let it be noted that the present point of view does away with the unappealing feature, in Page's system, that Euler's formula

$$
e^{i x}=\cos x+i \sin x \quad\left(i^{2}=-1\right)
$$

is dimensionally nonhomogeneous, and that therefore dimensional homogeneity is not preserved when the

\footnotetext{
*Present address, General Dynamics, Fort Worth. Texas.
1 C. H. Page, J. Research NBS 65B (Math, and Math. Phys.) No. 4, 227-235 (1961).

${ }_{2}^{1961)}$ J. Palacios, Analyse dimensionnelle (Gauthier-Villars, Paris, 1960).

3 W. C. Michels and A. L. Patterson, Elements of Modern Physics (Van Nostrand, Princeton, N.J., 1951), namely section 13.4.

4 F. Häberli, Schweiz. Arch. angew. Wiss. Techn. 15, 343 (1949).

5 P. Moon and D. E. Spencer, J. Franklin Inst. 248, 495 (1949).

${ }^{6}$ International Organization for Standardization, Doc. ISO/TC 12, 131 E, December, 1954 .
}

complex exponential formalism is used in physics instead of trigonometric functions. Of course, the difficulty can be disposed of by stating that "this is an illustration of the fact that equations used in physics need not be dimensionally homogeneous when terms arise from the use of an artifice. The function $e^{j \theta}$ does not actually occur in physics; it is introduced with the convention that its real part alone (or its imaginary part alone) represents a variable of interest. Nonartificial exponentials, such as $e^{-W / k T}$, have numeric exponents." (See footnote 1.) But it seems simpler to define the angular dimension so that Euler's formula is dimensionally homogeneous; that will be done presently. (However, the possibility of reconciling Page's scheme with the requirement of homogen eity will be shown at the end of section 3.) Moreover, the present treatment seems to allow a more straightforward extension to solid angles, taken as another distinct fundamental quantity, on the same footing with plane angle, by simply substituting "steradian" for "radian" and "area" for "length" in eq (1a).

On the other hand, this attempt misses the pleasant feature, in Page's work, that torque and energy acquire different dimensions (as do action and angular momentum, or areal velocity and kinematic viscosity coefficient). But there really is no reason, in the theory of dimensions, to demand that no two different physical quantities should have the same dimensional formula; and it seems hardly possible to devise a dimensional system in which no such duplication would happen anywhere: for instance, even in Page's system, a frequency and a velocity gradient have the same dimensions.

\section{Basic Definitions}

The appearance of angles in an equation can often be traced to the relation

$$
s=\theta R
$$

between an are $s$ of a circle, its radius $R$ and the central angle $\theta$ measured in radians. The necessity of dimensional homogeneity of both sides of eq (1) (in which $s$ and $R$ are lengths) seems to imply that $\theta$ is dimensionless. 
It has been tried (see footnote 6 ) to avoid contradiction by taking into consideration two kinds of "angles": the "geometrical angle" which is the usual one, a quantity related to the portion of the plane contained between two concurrent straight lines, and the "analytical angle" which is the $\theta$ to be found in eq (1). But the so-called "analytical angle" is no new notion indeed: it is plainly the measure of the angle in radians. This can be seen immediately from eq (1), which originates in a theorem stating that in a circle two arcs are proportional to their central angles. When one of the ares is equal to the radius its central angle is one radian, and the proportion becomes

$$
s=(\theta / 1 \mathrm{rad}) R,
$$

which is the correct form of eq.(1), with $\theta$ a true "geometrical" angle.

How is it that one should think himself obliged to particularize the measures of angles under the name of "analytical angles," while not feeling any need whatsoever of "analytical lengths," "analytical masses" or "analytical time"? The explanation, and the reason why mechanics could be developed without recognizing the fundamental dimension of angle, is that angles often appear in mechanics through their measure in radians, which indeed can be seen to be derived from eq (1a). Something similar happens in other chapters of physics: for instance it is well-known that the dimensional analysis in photometry is less complete if the further fundamental dimension of solid angle is not taken into account; actually what is here being said of angles can be extended to solid angles.

The basic angular quantity will be taken here to mean the ordinary "geometrical" angle, certainly the most primary notion in any study of angles. 'Trigonometric functions will be defined in the elementary way as ratios of lengths, i.e., dimensionless quantities. This is simpler than starting from power expansions, and has the advantage of putting sin and $c o s$ on an equal footing.

Since the denominator (1 rad) will often appear, I shall write for the sake of brevity, for any quantity $Q$ :

$$
Q /(1 \mathrm{rad})=\langle Q\rangle \text {. }
$$

This should not be mistaken for the dimensionless measure of $Q$ : it is so only for angles. (When $Q$ is an angle $\theta,\langle\theta\rangle$ is simply the "analytical" alternative to the "geometrical" angle $\theta$.) "Thus eq (1a) will be written

$$
s=\langle\theta\rangle R .
$$

\section{Angular Dimension in Mathematics}

Restrictions are met in mathematics about angles, at least on two occasions: these are eq (1), and the following:

$$
\lim _{\theta \rightarrow 0}(\sin \theta) / \theta=1,
$$

both of which are only true when $\theta$ is measured in radians. Equation (1) has just been dealt with, and its general form (3) has been obtained. The same can be done to eq (4), which is a direct daughter of eq (1). The reader should turn back to the elementary demonstration of eq (4); the general scheme is: (1) show that the length of an are $\theta$ of a circle lies between those of two line segments which are proportional to $\sin \theta$ and $\tan \theta ;(2)$ divide by the radius $R$ : the two segments yield $\sin \theta$ and $\tan \theta$, while the arc becomes $s / R$, or $\langle\theta\rangle$ on account of eq (3). Thus, the true form of eq (4), valid for any unit of angle, is

$$
\lim _{\theta \rightarrow 0}(\sin \theta) /\langle\theta\rangle=1 .
$$

A similar equation would be obtained with $\tan \theta$ instead of $\sin \theta$.

The equations showing how to replace the sine and tangent of a small angle by the angle itself follow in the correct form

$$
\sin \theta \approx\langle\theta\rangle ; \tan \theta \approx\langle\theta\rangle .
$$

As a consequence, every equation derived from eq (3) or (5) should be recalculated. The details of the calculations are left to the reader.

Here are a few results:

(a) Derivatives of trigonometric functions:

$$
d(\sin x) / d x=(1 / 1 \mathrm{rad}) \cos x,
$$

or

$$
d(\sin x)=\cos x d\langle x\rangle,
$$

and analogous expressions for the derivatives of cos $x, \tan x, \ldots$ An application of eq (7) which will be of further use is

$$
d(\sin \omega t) / d t=\langle\omega\rangle \cos \omega t .
$$

(b) By comparing the power expansions of $e^{i x}$ to those of $\cos x, \sin x$ derived from eq (7), namely

$$
\begin{gathered}
\cos x=1-\frac{1}{2}\langle x\rangle^{2}+(1 / 4 !)\langle x\rangle^{4}-\ldots, \\
\sin x=\langle x\rangle-(1 / 3 !)\langle x\rangle^{3}+\ldots,
\end{gathered}
$$

one readily obtains for Euler's equation the dimensionally homogeneous form

$$
e^{i<x>}=\cos x+i \sin x,
$$

which will be used in the form

$$
e^{i<\omega>t}=\cos \omega t+i \sin \omega t .
$$

From eq (10) follows

$$
\cosh i\langle x\rangle=\cos x ; \sinh i\langle x\rangle=i \sin x .
$$

(c) When remembering eq (7) and computing again the following integral, one gets

$$
\begin{aligned}
\int\left(a^{2}-y^{2}\right)^{-1 / 2} d y & =(1 / 1 \mathrm{rad}) \sin ^{-1}(y / a)+C \\
& =\left\langle\sin ^{-1}(y / a)>+C,\right.
\end{aligned}
$$

and similar expressions for $\cos ^{-1}$ and $\tan ^{-1}$. 
It can be noticed that all the above equations are homogeneous with respect to dimensions ( $x$ is an angle, $t$ the time, $y$ and $a$ any quantities of the same dimension; $\omega$ has the dimension $\theta T^{-1}$, if $\theta$ denotes the angular dimension): the arguments of trigonometric functions are angles, but those functions themselves are pure dimensionless numbers, as well as the arguments of hyperbolic functions and the exponents. With those equations in mind no difficulty can be experienced in setting up the equations of mechanies.

Before switching to mechanical equations, let it be pointed out that homogeneity can easily be restored in Page's scheme for the equation

$$
e^{i x}=\cos x+i \sin x,
$$

and more generally for the polar expression of any complex number, wherein the same problem occurs.

What needs be done is simply assigning the dimension of angle to the complex " $i$ ". Since $[\theta]^{2}$ $=[1]$ (Page's notation), there is no conflict with

$$
i^{2}=-1
$$

as a quantity equation; and as $\sin x$, in that scheme, also has the dimension of angle, that would make both the exponent $i x$ and the product $i \sin x$ dimensionless.

The unusual feature is that in any complex number $a+b i, a$ is a numeric but $b$ has the dimension of angle (or more generally $a$ has the dimensions of the modulus and $b$ those of modulus times angle). The appearance of an angular dimension in complex number representation would not seem incongruous if one remembers that the natural geometric representation of complex numbers is in a plane.

But the feature just alluded to implies a further consequence. If homogeneity is to be preserved throughout, the imaginary part $b$ of any complex number $a+b i$ must have the dimension of angle, even when $b=1$. This implies introducing some arbitrary "unit of imaginary part," say $u$, with the dimension of angle, by means of which the complex number $(a+i)$ can be written $(a+u i)$. It is then obvious that the use of $u$ is completely equivalent to putting

$$
j=u i
$$

(j dimensionless) and writing

$$
a+b i=a+(b / u) j \text {. }
$$

Now the dimensionless $b / u$ is exactly the expression defined by eq (2). In other words, in the suggested "homogenized Page's formalism," the equations involving complex numbers go over into a form equivalent to that of the present approach.

The comparison between Page's scheme and the present proposal can thus be summarized as follows:

In Page's scheme the relations

$$
d(\sin x) / d x=\cos x ; e^{i x}=\cos x+i \sin x
$$

are quantity equations, whereas in the present proposal eqs (8) and (10) include explicit reference to the radian. But this advantage of Page's scheme is, to this writer's feeling, canceled by the necessity of either introducing $u$ in the complex numbers or retaining inhomogeneous equations wherever complex numbers are involved in the polar form.

The only irreducible difference between the two approaches boils down to the fact that in Page's scheme the dimension of angle is singled out by the requirement that $[\theta]^{2}=[1]$, while all four dimensions, as such, are put on an equal footing in the present approach. (In fact, however, the explicit reference to the radian, or to $u$, also singles out the angular dimension in both systems.) Admittedly, this is essentially a matter of taste.

\section{Angular Dimension in Kinematics}

The basic appearance of angles in kinematics is through angular velocity. The mutual dependence of linear and angular velocities in a circular motion is obtained by differentiating eq (3) with respect to time, and putting $v$ for $d s / d t$ and $\omega$ for $d \theta / d t$ :

$$
v=\langle\omega\rangle R \text {. }
$$

This equation is dimensionally homogeneous, for the dimensional formula of $\omega$ is $\theta T^{-1}$; thus that of $\langle\omega\rangle$ is $T^{-1}$.

Let us now investigate a few consequences of eq (12). From eq (12) the dimensionally correct expressions can be deduced for the rotational kinetic energy of a solid:

$$
T=\sum \frac{1}{2} m v^{2}=\frac{1}{2} I\langle\omega\rangle^{2},
$$

for the centrifugal force on a particle:

$$
F_{c}=m\langle\omega\rangle^{2} R
$$

and for the law of areas:

$$
R^{2} d<\theta>/ \vec{i} t=C .
$$

The angular velocity vector $\boldsymbol{\Omega}$ of a rotating solid is defined by the equation

$$
\mathbf{v}=\boldsymbol{\Omega} \times \mathbf{r} .
$$

It is usually considered to have a length equal to the value of the angular velocity $\omega$. The dimensional homogeneity of eq (13) (where the crossproduct symbol is dimensionless) shows that the true relation, when angle is considered a fundamental quantity, is

$$
\Omega=\langle\omega\rangle \text {, }
$$

with the dimensional formula $T^{-1}$. This dimensional formula is found to agree with the particular case of the vortex vector of a fluid:

$$
\mathbf{\Omega}=\frac{1}{2} \nabla \times \mathbf{v},
$$

in which no angular dimension appears. 
Binet's formula expressing the radial acceleration of a point obeying the law of areas reads

$$
\left.j_{r}=-\left(A^{2} / r^{2}\right) \cdot\left[d^{2}(1 / r) / d<\theta\right\rangle^{2}+1 / r\right],
$$

where $A$ is the constant of area.

And, finally, the radius of curvature $R$ of a curve in a point is defined, in terms of the infinitesimal arc $d s$ and the corresponding angular displacement $d \theta$ of the tangent, by

$$
R=d s / d\langle\theta\rangle,
$$

which is a direct application of eq (3).

\section{Angular Dimension in Dynamics}

The appearance of angles in dynamics may derive from many of the above equations:

From eq (6) comes the proper form of the equation yielding the shear angle:

$$
\langle\psi\rangle=2 T(1+\sigma) / E
$$

(where $\sigma$ is Poisson's ratio and E Young's modulus), and the physical expression of the nondiagonal components $\epsilon_{i j}$ of the infinitesimal strain tensor of a continuous medium in terms of the shear angles relevant to the directions $i, j$ :

$$
\epsilon_{i j}=\left\langle\psi_{i j}\right\rangle \text {. }
$$

Also from eq (6) follows the second-order approximation of the formula for the period of the circular pendulum with amplitude $\theta$ :

$$
\tau=2 \pi(l / g)^{1 / 2}\left(1+\langle\theta\rangle^{2} / 16+\ldots\right) .
$$

Equation (9) leads to the expression of the equation for the harmonic motion of a point on a straight line under a central attractive force $-k r$ proportional to distance:

with

$$
x=\left(v_{0} /\langle\omega\rangle\right) \sin \omega t+x_{0} \cos \omega t,
$$

$$
\langle\omega\rangle=(k / m)^{1 / 2} ;
$$

the period is

$$
\tau=2 \pi /\langle\omega\rangle
$$

and has the correct dimension of a time.

The analogous motion under a central repulsive force $+k r$ is deduced from eq (17) by changing $k$ into $-k$ in eq (17) and keeping eq (18) as a definition of $\omega$; it reads, thanks to eq (11):

$$
x=x_{0} \cosh \langle\omega\rangle t+\left(v_{0} /\langle\omega\rangle\right) \sinh \langle\omega\rangle t .
$$

Equations (13), (14) show that the relation between the (normal) torque $\mathfrak{M}$ on the axis causing a gyroscope to precess, the moment of inertia $I$ about that axis, and the angular velocities of rotation and of precession is

$$
\mathfrak{M}=\langle\omega\rangle\left\langle\omega^{\prime}\right\rangle I,
$$

where $\mathfrak{M}$ has the conventional dimension.

Equation (15) is responsible for the formulation of the law of distribution of tension in a string constrained to lie on a surface with friction, in terms of the angle $\theta$ of rotation of the tangent:

$$
T=T_{0} e^{f<\theta>} .
$$

The same eq (15) would be involved in the study of the motion of a point missile in the air with friction.

The dimensional homogeneity shows that the relation between torque, angle of rotation, and the torsional constant in a twisted wire is

$$
\mathfrak{M}=C\langle\theta\rangle \text {, }
$$

which can also be seen theoretically to proceed from eq (16). The expression for the torsional period follows with the correct dimension:

$$
\tau=2 \pi(I / C)^{1 / 2}=2 \pi(I<\theta>/ \mathfrak{M})^{1 / 2} .
$$

\section{Conclusion}

The above survey does not claim to be anything like an exhaustive study of the introduction of an angular dimension in mechanics. It is just a collection of examples to show that no disadvantage can arise from its systematic use. Actually, this writer taught rational mechanics for five years with this system of dimensions without ever coming across any inconvenience. ${ }^{7}$ On the contrary, he found the new point of view often illuminating since it allows an appreciable widening of the scope of dimensional analysis.

7 J. E. Romain, Publications de l'Université de l'Etat, Elisabethville (Katanga), No. 3,1962 . 